\title{
ULTRASOUND-GUIDED BRACHIAL PLEXUS NERVE BLOCK IN DONKEYS
}

\author{
Ayman S. Atiba ${ }^{1 *}$, Alaa Ghazy ${ }^{1}$, Foad A. Farrag², Mohammed A. El-Magd², \\ Essam A. Almadaly ${ }^{3}$
}

${ }^{1}$ Departments of Surgery, Anesthesiology and Radiology, Faculty of Veterinary Medicine, Kafrelsheikh University, 33516, Egypt, ${ }^{2}$ Department of Anatomy and Embryology, Faculty of Veterinary Medicine, Kafrelsheikh University, 33516, Egypt, ${ }^{3}$ Department of Theriogenology, Faculty of Veterinary Medicine, Kafrelsheikh University, Egypt

*Corresponding author, E-mail: atiba_2003@yahoo.co.uk

\begin{abstract}
This study was conducted to describe and evaluate the ultrasound-guided technique and the anatomical relation for brachial plexus block in donkeys. In the first study, three donkey's cadavers were used to determine the neuroanatomical description of the brachial plexus. In the second study, four donkeys were used to evaluate the ultrasound structure of brachial plexus and the distribution of methylene blue solution that was injected around the nerves during subsequent postmortem anatomical dissection. In the third study, six donkeys were used to evaluate the efficient of the ultrasound-guided brachial plexus block after injection of lidocaine. Ultrasound was used to identify brachial plexus and blood vessels. Anatomical scrutiny assured the relationship between the nerves and the blood vessels. The nerves were adequately stained with methylene blue solution in all donkeys. A volume of $25 \mathrm{~mL}$ of lidocaine per injection site resulted in effective sensitive and motor block of forelimbs nerves after brachial plexus block. Based on the obtained data we could conclude that brachial plexus nerve block guided by ultrasound can be used in surgery of donkey distal fore limb.
\end{abstract}

Key words: ultrasound; brachial plexus; nerve block; donkey

\section{Introduction}

The donkey (Equus asinus) is the most important working animal in many parts of the world (1). Donkeys provide one of the most cost effective sources of transport in the rural agricultural sector. There are an estimated 59 millions of donkeys and mules in the world (2). Although greater populations of donkeys live in developing countries, very little scientific information specific for this species exists in the literature.
Peripheral nerve block techniques are becoming increasingly popular for performing certain surgeries in both animals and humans (3-5). These techniques do not only decrease general anesthesia drawbacks but they also lead to effective analgesia (6-8). In animals many surgical operations especially in the distal parts of the limbs can be performed under peripheral nerve block such as sciatic and femoral nerves block of the pelvic limbs in cattle, sheep and dogs (9-11) and brachial plexus nerve block in the forelimbs in cattle, dogs and cats $(3,5,7,9)$. 
In humans, brachial plexus block is very universal and safe, and allows anesthesia for the upper limb. Therefore, various operations, principally orthopedic, could be carried out under this block $(12,13)$.

Several techniques exist to produce local anesthetic blockade of the brachial plexus include the use of anatomic landmarks (blind technique), a nerve locator technique, and ultrasound guidance technique. Many studies comparing these techniques and all stated that ultrasound-guided one is the most effective technique to facilitate the peripheral nerve block $(8$, $14,15)$.

Previous literatures lack detailed description for neuroanatomy and block technique of brachial plexus in donkeys. Thus, this study was designed to describe and evaluate the ultrasound-guided technique and the anatomical relation for brachial plexus block in donkeys.

\section{Materials and methods}

The experimental protocol was approved by the Animal Care and Use Committee of Faculty of Veterinary Medicine, Kafrelsheikh University.

\section{Study 1: Anatomical study of the brachial plexus}

For the anatomical dissection of the brachial plexus, cadavers of three donkeys $(23 \pm 2$ months old, weighing $167 \pm 10 \mathrm{~kg}$ ) were used. The forelimb of the three donkeys were separated carefully from the trunk by cutting the skin, the pectoralis superficialis and pectoralis profundus muscles at their origin on the sternum, brachiocephalicus and trapezius muscles at the cranial border of scapula, serratus ventralis, rhomboideus muscles at their insertion near the dorsal border of scapula and latissmus dorsi muscle at the caudal border of scapula. The forelimb was abducted to expose the axillary artery and brachial plexus. The nerves and main blood vessels were dissected and identified. Finally, the dissected region was photographed.

Study 2: Ultrasound-guided brachial plexus blockade in cadavers

The ultrasound images of the brachial plexus blockade of both forelimbs from four donkeys
(18 \pm 4 months old, weighing $181 \pm 18 \mathrm{~kg}$ ) were taken using a high frequency $5-10 \mathrm{MHz}$ linear transducer (Mindray Diagnostic Ultrasound System, Z5, Germany). The transducer was placed in front of the cranial border of scapula and the first rib and dorsal to the shoulder joint (Fig. 1C). Color Doppler mode was utilized to aid vessels identification. The location of brachial plexus nerves were injected with $25 \mathrm{~mL}$ methylene blue (Sigma-Aldrich, USA) using a 20 gauge, $90 \mathrm{~mm}$ spinal needle. The needle was inserted just ventral to the ultrasound transducer and vertical to the nerves then methylene blue was injected when the needle tip reached a depth of $1 \mathrm{~mm}$. After injection, donkeys were euthanized and injection site was dissected to determine staining distributions around nerves.

Study 3: Ultrasound-guided brachial plexus blockade in live donkeys

For the ultrasound-guided brachial plexus blockade, six donkeys (aged $19 \pm 7$ months and weighing $188 \pm 41 \mathrm{~kg}$ ) were used. The brachial plexus nerves were blocked unilaterally. Each donkey was used for three times with 1 week interval. The donkeys were mild sedated with $0.3 \mathrm{mg} / \mathrm{kg}$ IV xylazine (Xyla-Ject, ADWIA Pharmaceuticals Co. Cairo, Egypt). In the standing position, the skin of the craniolateral aspect of the first rib was clipped. The brachial plexus were blocked with $25 \mathrm{ml}$ of a lidocaine (Debocaine 2\% Al-Debeiky Pharmaceutical Industries Co., Egypt). To evaluate analgesia, a pin-prick test was applied with a 21 gauge needle inserted through the skin. The sensitive response was scored using a three-point numerical rating scale (Table 1). The areas of sensitive responses were evaluated according to the sensory branches of brachial plexus (Table 2).

The motor effect was evaluated as previously described (3) and was scored using a three-point numerical rating scale (Table 1). The sensitive and motor responses of the forelimb were evaluated and compared before and after lidocaine injection. Twelve injections were given with $10 \mathrm{~min}$ intervals. Moreover, each injected forelimb was compared with the other contralateral non-injected forelimb. 


\section{Statistical analysis}

Descriptive statistical data are expressed as medians and ranges. The effects of local anesthetic on brachial plexus in the various anatomical regions were analyzed by the non-parametric Friedman test with pairwise comparisons using SPSS for Windows Version 16 (SPSS Inc., IL, USA). A value of $\mathrm{P}<0.05$ was considered significant.

\section{Results}

Study 1: Anatomical study and dissection of the brachial plexus nerves

The gross dissection of donkeys brachial plexus revealed that it was composed of the anastomosis of the ventral branches of the last three cervical $\left(6^{\text {th }}, 7^{\text {th }}\right.$ and $\left.8^{\text {th }}\right)$ and the first two thoracic spinal nerves. The brachial plexus was located cranial to the first rib and dorsal to scalenius medius muscle. It was covered laterally by subclavius and supraspinatus muscles slightly above the shoulder joint (Figs. 1A and B). The brachial plexus gave many branches which innervated the skin and muscles of forelimb in addition to parts of the lateral wall of thorax and abdomen as well as parts of the shoulder girdle (Fig 1B). The branches of brachial plexus either motor or sensory were summarized in table 2.

\section{Study 2 and 3: Ultrasound-guided nerve blockade}

The brachial plexus was identified as a hyperechoic band with several intermittent hypoechoic strips separated by hypoechoic lines (Figs. 2A-C). These hyperechoic structures were confirmed to be brachial plexus after utilizing color Doppler mode, which showed the axillary blood vessels ventral to these hyperechoic structures (Fig. 2B). The ultrasound transducer was placed cranially near the first rib. In this position the needle was advanced above the brachial plexus and axillary blood vessels. After assuring that blood was not withdrawn, methylene blue was injected. The extent of dye was monitored as an anechoic area proximal to the brachial plexus. Staining of brachial plexus was observed in all donkeys. The dye was spread more than $2 \mathrm{~cm}$ in 3 donkeys (Fig. 1D). During this procedure, only one animal was intravascularly injected in axillary vessels.

Figs. 2D and E show the scores of the sensitive and motor effects after ultrasound-guided brachial plexus block in 16 forelimbs. One animal was died after 1 week with a reason not related to the current study. The sensory score for the blocked area showed a significant increase at 30, 40 and $50 \mathrm{~min}$ in musclocutaneous nerve area, at 30 and $40 \mathrm{~min}$ for the area of median nerve, and at $30 \mathrm{~min}$ for the area of ulnar nerve $(p<0.05)$. The motor scores increased significantly at time points $20,30,40$ and $50 \mathrm{~min}$. A motor effect was extended more than the sensory effect in all examined animals.

\section{Discussion}

To the best of our knowledge, this may be the first study to investigate the ultrasoundguided brachial plexus blocking in donkeys. With this study we were able to demonstrate the feasibility to block the brachial plexus in donkeys with a highly successful rate.

Table 1: The score of sensitive and motor response (3)

\begin{tabular}{lll}
\hline Score & Sensitive response & Motor response \\
\hline 0 & Normal response & $\begin{array}{l}\text { Normal gait while walking and no abnormal } \\
\text { clinical sign while standing. } \\
\text { Donkey can walk while bearing mild to moder- } \\
\text { ate weight and shows no abnormal sign while } \\
\text { standing. } \\
\text { Donkey can walk but is barely able to bear } \\
\text { weight while walking, and shows abnormal } \\
\text { signs while standing. }\end{array}$ \\
\hline
\end{tabular}


Table 2: Summary of branches of brachial plexus and its motor and sensory innervation

\begin{tabular}{|c|c|c|}
\hline \multirow[t]{2}{*}{ Branches of brachial plexus } & \multicolumn{2}{|c|}{ Innervation } \\
\hline & Motor & Sensory \\
\hline Long thoracic nerve & $\begin{array}{l}\text { Thoracic part of serratus ventra- } \\
\text { lis muscle. }\end{array}$ & \\
\hline Dorsal thoracic nerve & Latissmus dorsi muscle. & \\
\hline Lateral thoracic nerve & Cutaneous trunci muscle & Skin of lateral thoracic wall \\
\hline Cranial pectoral nerve & $\begin{array}{l}\text { Superficial pectoral and subcla- } \\
\text { vian muscles }\end{array}$ & \\
\hline Caudal pectoral nerve & Deep pectoral muscles & \\
\hline Suprascapular nerve & $\begin{array}{l}\text { Supra spinatus and infra spina- } \\
\text { tus muscles }\end{array}$ & \\
\hline Axillary nerve & $\begin{array}{l}\text { Deltoid, teres major, teres mi- } \\
\text { nor, capsularis humeri and } \\
\text { cleidobrachialis muscles }\end{array}$ & $\begin{array}{l}\text { Skin of craniolateral aspect } \\
\text { of brachium and cranial as- } \\
\text { pect of antebrachium }\end{array}$ \\
\hline Subscapular nerve & Subscapularis muscle & \\
\hline Musculocutaneous nerve & $\begin{array}{l}\text { Coracobrachialis, brachialis } \\
\text { and biceps brachii muscles }\end{array}$ & $\begin{array}{l}\text { Skin of medial part of an- } \\
\text { tebrachium }\end{array}$ \\
\hline Radial nerve & $\begin{array}{l}\text { Triceps brachi, anconeus, ten- } \\
\text { sor fascia antebrachii, extensor } \\
\text { carpi radialis, common digital } \\
\text { extensor, lateral digital exten- } \\
\text { sor, extensor carpi ulnaris and } \\
\text { extensor carpi obliqus }\end{array}$ & $\begin{array}{l}\text { Skin of the lateral aspect of } \\
\text { brachium and antebrachium }\end{array}$ \\
\hline Ulnar nerve & $\begin{array}{l}\text { Flexor Carpi Ulnaris, superfi- } \\
\text { cial digital flexor and ulnar } \\
\text { head of Flexor Digitorum Pro- } \\
\text { fundus, interosseous muscles }\end{array}$ & $\begin{array}{l}\text { Skin of the caudal aspect of } \\
\text { antebrachium and dorsolat- } \\
\text { eral aspect of metacarpus } \\
\text { and digit. }\end{array}$ \\
\hline Median nerve & $\begin{array}{l}\text { Flexor carpi radialis, humoral } \\
\text { and radial head of deep digital } \\
\text { flexor muscles }\end{array}$ & $\begin{array}{l}\text { Skin of the palmar aspect of } \\
\text { metacarpus and digit. }\end{array}$ \\
\hline
\end{tabular}

The detailed description of Equine forelimb nerve supply has been found in several standard anatomical textbooks (16-19). Briefly, this nerve supply comes from the brachial plexus which is a complex network of nerves formed by the anastomoses of the ventral roots of some spinal nerves extending from the $6^{\text {th }}$ cervical nerve to the $2^{\text {nd }}$ thoracic nerve. It passes between the two parts of the scalenius medius muscle and the medial aspect of shoulder in the axillary region. The major nerves emanated from the brachial plexus are the suprascapular, subscapular, musculocutaneous, axillary, radial, median, and ulnar nerves. Brachial plexus supplies sensory and motor innervation of the forelimb (20). The previous description of the nerve supply of forelimb in horses coincides with the findings of the current study regarding the brachial plexus position and nerves emanating from it in donkeys. The higher degree of similarity between donkeys and horses might be attributed to close relation between the two species which belong to the same family (Equidae) and genus (Equus). 

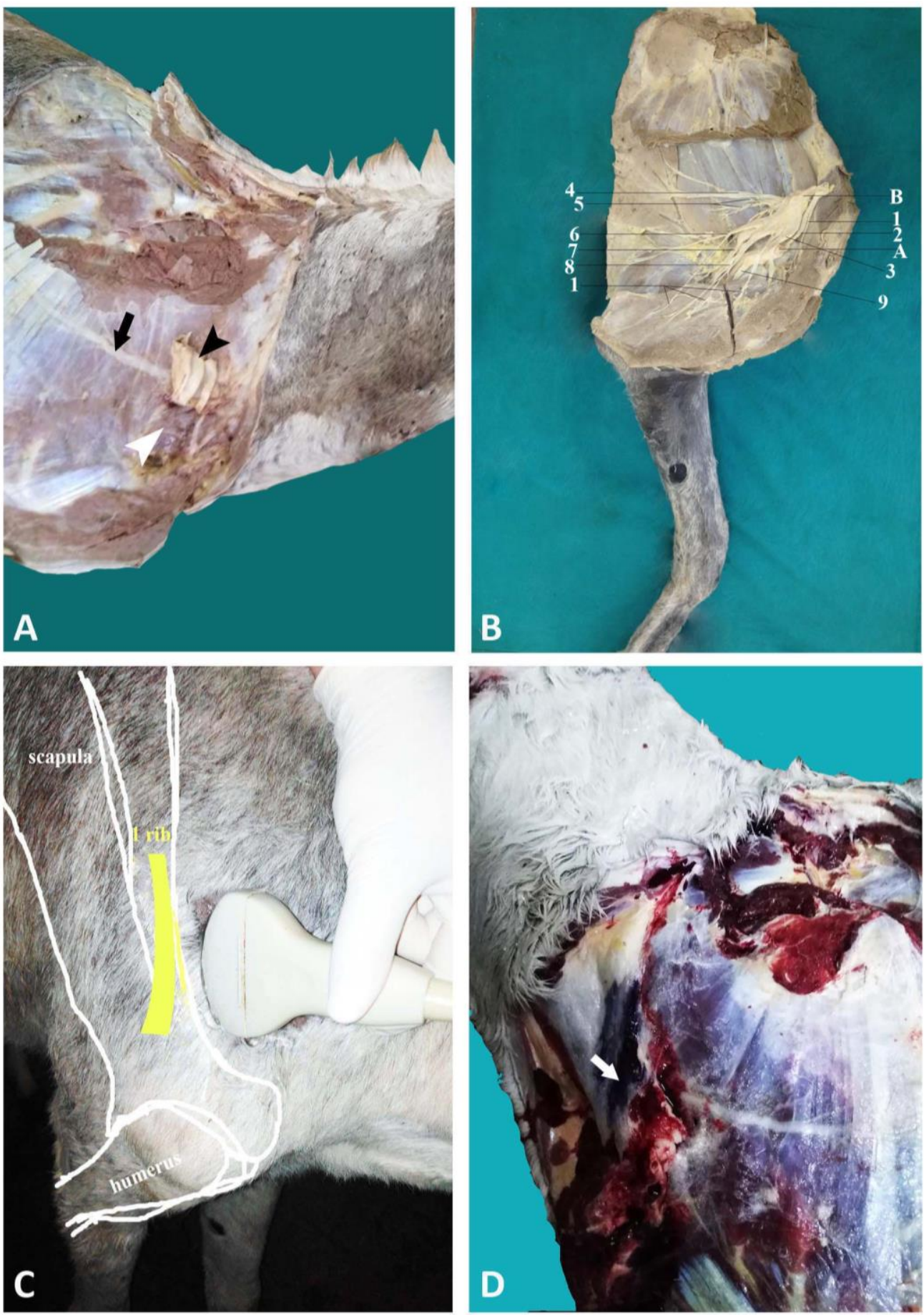

Figure 1: Position, branches of brachial plexus and site of ultrasound transducer. A. Position of brachial plexus (black arrowhead), scalenius medius muscle (white arrowhead) and long thoracic nerve (black arrow). B. Axillary artery (A) surrounded by branches of brachial plexus (B); pectoral nerves (1), musculocutaneous nerve (2), median nerve (3), dorsal thoracic nerve (4), subscapular nerve (5), lateral thoracic nerve (6), radial nerve (7), ulnar nerve (8) and the musculocutaneous with median nerves (9). C. Position of ultrasound transducer in front of cranial border of scapula and first rib and dorsal to the shoulder joint. D. Site of injection of methylene blue dye (white arrow) in the region of brachial plexus 


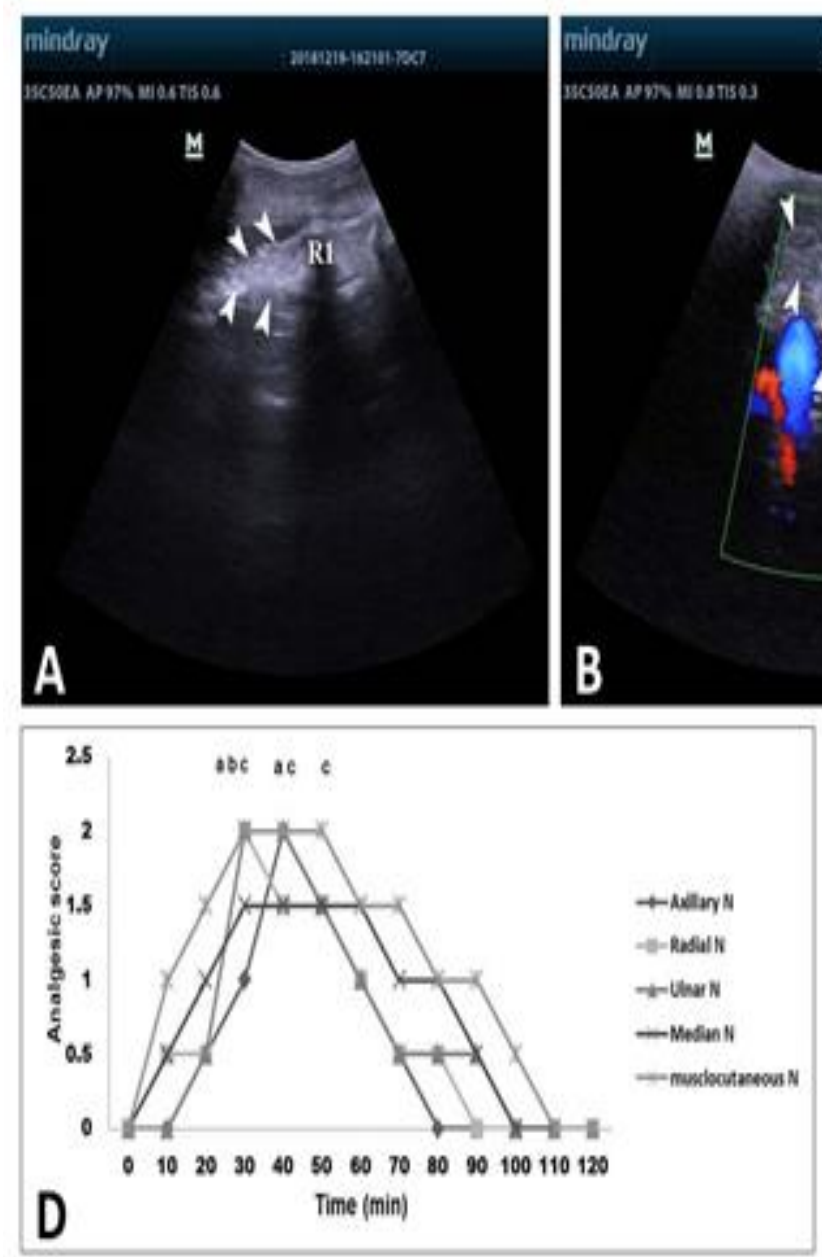

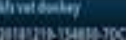

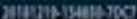

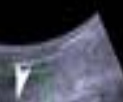

RI

4.
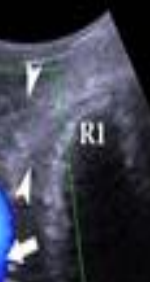
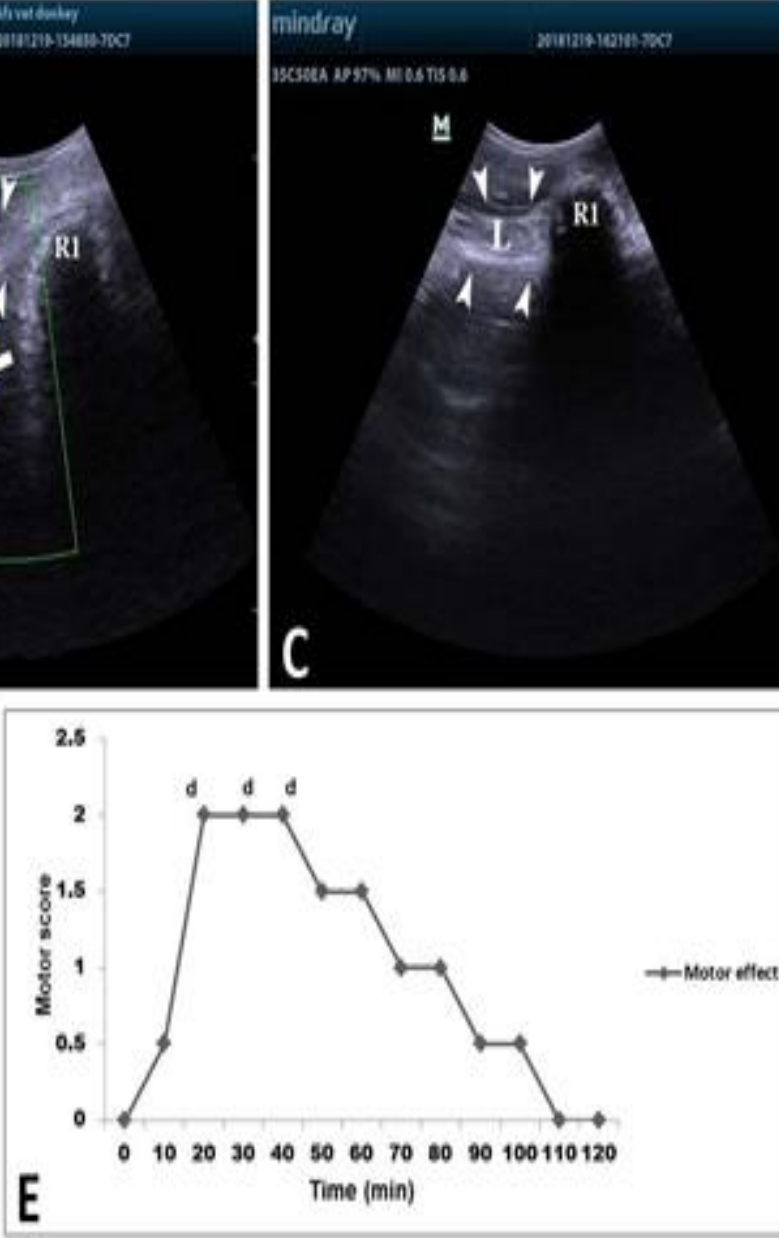

Figure 2: Ultrasound images of the area around the brachial plexus, the sensory and motor score after nerve block. A. Ultrasound image of the brachial plexus (the area surrounded by the arrowheads) and the first rib (R1). B. Color Doppler ultrasound image of the brachial plexus (the area surrounded by the arrowheads) and the axillary blood vessels (arrow). C. Ultrasound image of the brachial plexus after injection of local anesthetic drug (L). D. Sensitive and motor (E) scores after ultrasound-guided brachial plexus block in donkeys. Data are expressed as the median. Symbols indicate significant differences from baseline value within a defined anatomical subarea $(\mathrm{P}<0.05)$

Currently, the use of the ultrasonography has been reported to provide an accurate guide for performance of peripheral nerve block when compared with the use of conventional blind or electrolocation techniques $(8,15)$. This technique provided the ability to administer a calculated amount of anesthetic drug at the target site under real-time observation $(7,14)$. Additionally, it minimized complications like maldistribution of local anesthetic or intravascular needle placement (21). In the present study, among all injected cases, only one case exhibited intravascular insertion of the needle.
Peripheral nerves may look hypoechoic or hyperechoic on ultrasound image (22). In the present study, the brachial plexus appeared hyperechoic structures dorsal to the axillary vessels. Similar findings were reported for ultrasound-guided nerve blockade of the brachial plexus in humans, calves, dogs and cats $(3,7,9$, 13, 15).

The brachial plexus provides the sensory and motor supply of the thoracic limb. Therefore, its successful block would provide complete desensitization of the whole forelimb $(7,20)$. In the present study, the regions dominated by 
musculocutaneous, median and ulnar nerves showed a significant sensory scores $(\mathrm{p}<0.05)$. While the other regions dominated by radial and axillary nerves showed insignificant increased score as compared to the basal line time. Motor score was increased significantly and extended more than the sensory effect. Our obtained sensory and motor scores were more effective than that obtained after brachial plexus block in calves (3). This may be attributed to the volume of local anesthetic drug used. In calves, the volume was $10 \mathrm{ml} 2 \%$ lidocaine while we used $25 \mathrm{ml} 2 \%$ lidocaine. Increasing the dose of local anesthetic associated with efficacy of peripheral nerve block (23).

\section{Conclusion}

To the best of our knowledge, this may be the first description to the anatomical and ultrasound-guided brachial plexus block in donkeys. The ultrasound-guided technique was found to be a feasible and safe method to provide adequate analgesia of the forelimb in donkeys but further trials would be needed to assess the usefulness and the applicability of this technique in the clinical cases.

\section{Conflict of interest}

The authors declare that they have no conflict of interest.

\section{References}

1 .Lizarraga I, Sumano H, and Brumbaugh G. Pharmacological and pharmacokinetic differences between donkeys and horses. Equine Veterinary Education 2004; 16 (2): 102-12.

2. Al-Salihi K, Farhat S. The Society for the Protection and Welfare of Donkeys and Mules in Egypt "SPWDME": An overview. MRSVA 2014; 3 (3), 1-5.

3. Iwamoto J, Yamagishi N, Sasaki K, Kim D, Devkota B, Furuhama K. A novel technique of ultrasound-guided brachial plexus block in calves. Research in veterinary science 2012; 93(3):146771.

4. Gallacher K, Santos L, Campoy L, Bezuidenhout A, Gilbert R. Development of a peripheral nerve stimulator-guided technique for equine pudendal nerve blockade. The Veterinary Journal 2016; 217:72-7.
5. Portela DA, Verdier N, Otero PE. Regional anesthetic techniques for the thoracic limb and thorax in small animals: A review of the literature and technique description. The Veterinary Journal 2018 ; 241: 8-19

6. Edmondson MA. Local and regional anesthesia in cattle. Veterinary Clinics of North America: Food Animal Practice 2008; 24(2): 211-26.

7. Ansón A, Laredo FG, Gil F, Soler M, Belda E, Agut A. Evaluation of an ultrasound-guided technique for axillary brachial plexus blockade in cats. Journal of feline medicine and surgery 2017;19 (2):146-52.

8. Hagag U and Tawfiek MG. Blind versus ultrasound-guided maxillary nerve block in donkeys. Veterinary anaesthesia and analgesia, 2018; 45(1): 103-10.

9. Campoy L, Bezuidenhout AJ, Gleed RD, Martin-Flores M, Raw RM, Santare CL. Ultrasoundguided approach for axillary brachial plexus, femoral nerve, and sciatic nerve blocks in dogs. Veterinary anaesthesia and analgesia 2010; 37(2):144-53.

10. Re M, Blanco-Murcia J, Villaescusa Fernandez A, De Gaspar Simon I, Gomez de Segura IA. Ultrasound-guided anaesthetic blockade of the pelvic limb in calves. Veterinary Journal 2014; 200 (3):434-9.

11. Waag S, Stoffel MH, Spadavecchia C, Eichenberger U, Rohrbach H. Ultrasound-guided block of sciatic and femoral nerves: an anatomical study. Laboratory animals 2014; 48(2):97-104.

12. Enneking FK, Chan V, Greger J, Hadzic A, Lang SA, Horlocker TT. Lower-extremity peripheral nerve blockade: essentials of our current understanding. Regional anesthesia and pain medicine 2005; 30(1):4-35.

13. Nowakowski P. Ultrasound guided axillary brachial plexus block. Part 1 Basic sonoanatomy. Anaesthesiol Intensive Ther 2015; 47 (4), 409-416.

14. Singh S, Goyal R, Upadhyay KK, Sethi N, Sharma RM, Sharma A. An evaluation of brachial plexus block using a nerve stimulator versus ultrasound guidance: A randomized controlled trial. Journal of anaesthesiology, clinical pharmacology 2015; 31(3):370.

15. Akasaka, M and Shimizu M. Comparison of ultrasound-and electrostimulation-guided nerve blocks of brachial plexus in dogs. Veterinary anaesthesia and analgesia 2017; 44(3): 625-35.

16. Dams R, Lapras M, Florio R, Barone R. On malformations following the injection of delta-hydrocortisone acetate into the embryonal chicken egg and their transmission by a sole survivor. Comptes 
rendus des seances de la Societe de biologie et de ses filiales $1964 ; 158: 740-2$.

17. Getty R, Sisson S, Grossman J. The anatomy of the domestic animals 1975; 1:739-1211.

18. Nickel R, Schummer A, Seiferle E. The anatomy of the domestic animals. The locomotor system of the domestic mammals 1986; 1:150: 289.

19. Dyce KM, Sack WO and Wensing CJG. Textbook of Veterinary Anatomy-E-Book. Elsevier Health Sciences 2009; 4(3): 830-4.

20. Magilton J, Getty R and Ghoshal N. A comparative morphological study of the brachial plexus of domestic animals (goat, sheep, ox, pig and horse). Iowa State J. Sci, 1968; 42(3): 245-79.

21. Koscielniak-Nielsen Z. Ultrasound-guided peripheral nerve blocks: what are the benefits? Acta Anaesthesiologica Scandinavica 2008; 52(6): 727-37.

22. Marhofer P, Greher M, and Kapral S. Ultrasound guidance in regional anaesthesia. British journal of anaesthesia 2004; 94(1): 7-17.

23. Skarda RT. Local and regional anesthesia in ruminants and swine. Veterinary Clinics: Food Animal Practice. 1996; 12 (3):579-26. 